\title{
Transhumance en République du Bénin : états des lieux et contraintes
}

\author{
Paolo LESSE ${ }^{1 *}$, Marcel R. B. HOUINATO ${ }^{1}$, Jonas DJENONTIN ${ }^{2}$, Hippolyte DOSSA ${ }^{1}$, \\ Bouraima $\mathrm{YABI}^{3}$, Ismael TOKO ${ }^{3}$, Brice TENTE $^{3}$ et Brice SINSIN $^{1}$ \\ ${ }^{1}$ Département de Production Animale, Faculté des Sciences Agronomiques \\ de l'Université d'Abomey-Calavi (Bénin), 01 BP 526 Cotonou, Bénin. \\ ${ }^{2}$ Département de Production Animale, Faculté d'Agronomie \\ de l'Université de Parakou, BP 123, Parakou, Bénin. \\ ${ }^{3}$ Département de Géographie, Faculté des Lettes, Arts et Sciences Humaines \\ de l'Université d'Abomey-Calavi 01 BP 526 Cotonou, Bénin. \\ *Auteur correspondant, E-mail: lessepaolo@gmail.com,Tel: 0022995352163
}

\section{RESUME}

L'élevage pastoral joue un rôle prépondérant dans l'économie des pays de l'Afrique tropicale. Au Bénin, l'élevage, surtout celui du gros bétail, est essentiellement transhumant. De nombreuses études et rencontres scientifiques se sont consacrées à sa connaissance en vue de résoudre les difficultés qu'elle génère et de nombreuses dispositions réglementaires ont été adoptées dans ce même objectif. Le but de la présente étude est de faire un état des lieux de ces différents travaux afin d'en sortir les contraintes auxquelles ce système est confronté. Pour ce faire, plusieurs documents qui ont traité de la transhumance au Bénin et dans la sous-région Ouest Africaine ont été consultés. Les données obtenues ont été synthétisées. Ensuite, une caractérisation de la transhumance a été faite. L'outil utilisé est la triangulation. Il ressort de l'étude que les premiers écrits sur la transhumance datent de 1905. Les résultats ont également permis de voir qu'elle est confrontée à des problèmes d'alimentation, de variabilités climatiques, des textes règlementaires, de conflits et de disponibilités des infrastructures pastorales. Il ressort également que la transhumance demeure sous la forte influence des systèmes de culture surtout pendant la sécheresse. Mais la contribution des systèmes de culture demeure insuffisante.

(c) 2015 International Formulae Group. All rights reserved.

Mots clés : Système d'élevage, audit, difficultés, alimentation, Afrique de l'Ouest.

\section{Transhumance in Republic of Benin: state of art and constraints}

\begin{abstract}
The pastoral breeding plays a preponderant role in the economy of the sub-Saharan Africa countries. In Benin, beef cattle breeding are essentially nomadic. Numerous studies and meeting focused on it in order to solving the difficulties it brings and numerous legal dispositions were adopted for this same target. This study aims at making the inventory of these different studies in order to bring out the constraints confronted by the
\end{abstract}


system. Several documents on transhumance in Benin and the region were consulted and the data synthesized. This synthesis allowed to characterizing the study area, then the historic and characterization of nowadays transhumance. The tools used are the triangulation. The study reveals that the first papers on transhumance were writing in 1905. Moreover, the results showed that transhumance is confronted with pasture, climate variability, the statutory texts, the conflicts and the availability of pastoral infrastructures. The study also showed that transhumance is still under the heavy influence of crops systems mainly the dry drought season. However, the contribution to the farming system is still inadequate.

(c) 2015 International Formulae Group. All rights reserved.

Keywords: West Africa, difficult, alimentation, audit, breeding

\section{INTRODUCTION}

L'élevage et l'agriculture sont les deux principales activités économiques des populations rurales en Afrique Subsaharienne. La contribution moyenne de l'élevage au Produit Intérieur Brut (PIB) agricole régional est de 44\% (FAO, 2010). Avec un cheptel bovin de plus de 60 millions de têtes, 160 millions de petits ruminants et 400 millions de têtes de volailles, la région Ouest africaine est une aire privilégiée pour l'élevage (CEDEAO, 2009). Malgré cette importance, les systèmes d'élevage au Sahel et en Afrique de l'Ouest (SAO) demeurent traditionnels, extensifs, avec une productivité liée à la disponibilité en pâturage et en eau, qui commande les mouvements de troupeaux et définit les modes de production nomades ou transhumants (Eboh et al., 2008). Le pastoralisme transhumant en Afrique de l'Ouest reste confronté à de graves contraintes qui menacent sa base productive. Il en est de même au Bénin où l'élevage est la seconde activité économique après l'agriculture (Dugué et al., 2003). Il y contribue pour 6,2\% au PIB (FAO, 2010). Au Bénin, l'élevage, surtout celui du gros bétail, est essentiellement transhumant avec environ 2.211.000 têtes de bovins et plus 1.678.000 têtes d'ovins/caprins (FAOSTAT, 2014). Plusieurs travaux ont porté sur les systèmes d'élevage pratiqués au Bénin. Certains ont estimé que les écosystèmes naturels subissent de fortes dégradations (Convers, 2002 ; Kagone et al.,
2006). L'essentiel du fourrage consommé par les bovins en saison sèche provient essentiellement des espèces fourragères ligneuses. Ainsi, la strate ligneuse qui constitue l'élément stable du couvert forestier est sérieusement perturbée (Brisso et al., 2007). D'autres ont signalé les conflits (Lesse, 2011) découlant de la mobilité des animaux. L'objectif du présent article est de faire un état des lieux sur la pratique de transhumance au Bénin et d'en ressortir les principales contraintes.

La revue documentaire est l'outil utilisé dans le cadre de ce travail. A cet effet, plusieurs documents à savoir les articles, les thèses, les mémoires et les rapports techniques qui ont traité de la transhumance au Bénin et dans la sous-région ont été consultés. Les données obtenues ont été synthétisées afin de faire la caractérisation du milieu d'étude, puis l'historique de la transhumance. Ensuite une caractérisation de la transhumance telle pratiquée de nos jours a été faite. L'outil utilisé est la triangulation pour vérifier les informations selon les différentes sources. Une analyse diachronique a également été faite pour mettre en évidence la gestion communautaire et administrative de la transhumance au Bénin. Le système d'élevage local a été étudié selon le modèle de Lhoste et al. (1999) basé sur trois pôles principaux : le pôle humain, l'environnement et les troupeaux. 


\section{HISTORIQUE DE LA TRANSHUMANCE AU BÉNIN}

La pratique de la transhumance au Bénin a démarré avec les migrations des populations Peules et continue d'ailleurs d'être pratiquée par ces dernières. Les notes sur l'élevage en "Ex-Dahomey" ont fait cas de la transhumance pour la première fois en 1905 à l'occasion du rapport sur une épidémie de la péripneumonie contagieuse bovine, que les animaux en transhumance dans la région de Ségbana ont contractée auprès des animaux venus du Boussa (Nigéria). Le rapport indiquait que "ces mouvements de transhumance sont une menace pour le cheptel "dahoméen", faut-il pour cela les supprimer ?" Aujourd'hui, encore l'on continue toujours d'en parler et de se poser la même question. D'après Pecaud (1912), les Peuls du Bénin ont en majorité originaires du Fouta Djallon. Leur installation au Bénin date de très longtemps selon les notes sur l'élevage en "ExDahomey » mais Lombard (1957) la situe au $\mathrm{XVIII}^{{ }^{\mathrm{ème}}}$ siècle. L'histoire de la pratique de la transhumance ne peut être dissociée de celles des coutumes des populations peules (Atchy, 1976). Avant les années 1970, les déplacements des pasteurs sahéliens vers les zones plus humides ont surtout été dus à la saturation de l'espace pastoral plus qu'aux sécheresses répétitives. L'apparition de la problématique climatique avec le déficit hydrique et la diminution du fourrage prend essor à partir des années 1970, entraînant des mouvements importants de pasteurs vers le sud sahélien (Bernus, 1995). Une des clefs pour comprendre ces réactions selon Renard (2010) est de prendre en compte la valeur symbolique et sociale du troupeau chez les peuls, mais aussi la vision qu'en ont les populations extérieures. Bernus (1995) constate que c'est à partir de 1980 que l'on trouve les premiers écrits scientifiques sur le phénomène de pluriactivité chez les éleveurs peuls, ainsi que sur celui de la sédentarisation.

\section{RÈGLEMENTATIONS EN MATIÈRE DE TRANSHUMANCE}

Les transhumances intra-pays et transfrontalières sont règlementées par un éventail de lois, décrets, arrêtés, décisions et règlements pris tant au niveau communal, national qu'international. Ces textes légalement établis sont sujets à de nombreuses confusions liées à leur contenu, à leur cohérence d'ensemble, à leur vulgarisation, au niveau de compréhension des acteurs, des applications qui en sont faites, de leur abrogation ou non (Djohy, 2010).

\section{Textes communaux}

Dans toutes les communes concernées par le phénomène de transhumance, les mairies de ces dernières prennent des arrêtés pour réglementer la transhumance sur leurs territoires. On peut citer: l'arrêté $\mathrm{N}^{\circ} 54 / 024 / \mathrm{MKDI} / \mathrm{SG} / \mathrm{SGA} / \mathrm{SA}$ du 27 juillet 2006, portant Création, Composition et Fonctionnement de Comité Communal de Transhumance de la Commune de Kandi; l'arrêté $\mathrm{N}^{\circ} 014 / \mathrm{M}-\mathrm{CKM} / \mathrm{SG} / \mathrm{BAGD}$ du $1 \mathrm{er}$ Octobre 2004 portant réglementation de la vaine pâture, de la garde des animaux domestiques et de la transhumance dans la commune de Karimama; l'arrêté N No16/MCKM/SG/BAGD du 20 octobre 2004, Portant Création, Attributions et Fonctionnement des Comités Communaux, d'Arrondissement et de Village, de Prévention et de Gestion des Conflits Eleveurs-Agriculteurs dans la commune de Karimama et l'arrêté $\mathrm{N}^{\circ} 54 / 024 / \mathrm{MOI} / \mathrm{SG} / \mathrm{SGA} / \mathrm{SA}$ du 16 septembre 2009, portant Création, Composition et Fonctionnement de Comité Communal de Transhumance de la Commune de Ouinhi

\section{Textes nationaux}

$\mathrm{Au}$ niveau national, les textes qui réglementent la transhumance sont: la loi n'87-013 du 21 septembre 1987 portant réglementation de la vaine pâture, de la garde des animaux domestiques et de la transhumance ; l'arrêté Interministériel 1992 $\mathrm{N}^{\circ}$ 010/MISAT/MDR/D-CAB du 20 janvier 1992, Portant Création, Organisation, 
Attributions et Fonctionnement des comités de transhumance en République du Bénin ; la loi $\mathrm{N}^{\circ}$ 93- 009 du 2 juillet 1993 Portant régime des forêts en République du Bénin et le Décret N 96- 271 du 2 juillet 1996 Portant Modalités d'Application de la Loi $N^{\circ} 93-009$ du 2 juillet 1993 ; l'arrêté 1994 $\mathrm{N}^{\circ} 0039 / \mathrm{MISAT} / \mathrm{MDR} / \mathrm{DGAR}$ portant organisation de la transhumance 1993-1994; la loi $\mathrm{N}^{\circ}$ 2002-016 du 18 octobre 2004, portant régime de la faune en République du Bénin (Sans décret d'application); l'arrêté Interministériel 2006N²176/MAEP/MSPCL/DCAB/SGM/DRH/DE/SA, du 07 juillet 2006 Rendant obligatoire et synchrone la vaccination des animaux contre la Septicémie Hémorragique et la Péripneumonie Contagieuse chez l'espèce bovine, sur toute l'étendue du territoire national et le décret $\mathrm{N}^{\circ}$ 2009-241 du 09 juin 2009 portant création du comité chargé de régler les problèmes récurrents de transhumance entre les éleveurs peulhs et les agriculteurs sur le territoire national.

\section{Textes régionaux}

Les textes règlementant la transhumance au niveau sous régional sont : la décision CL/DEC.618 (XVIII) relative à la Politique cadre de l'UA sur le pastoralisme adoptée en 2011 ; la décision A/DEC.5/10/98 relative à la réglementation de la transhumance entre les Etats membres de la CEDEAO (issue de la 21ème session ordinaire de la conférence des chefs d'Etat et de Gouvernement tenue à Abuja du 30 au 31 octobre 1998), le règlement C/REG.3/01/03 relatif à la mise en œuvre de la réglementation de la transhumance entre les Etats membres de la CEDEAO (édicté lors de la quaranteneuvième session du conseil des ministres tenue à Dakar du 26 au 28 janvier 2003) pour assurer la mise en œuvre effective de la Décision A/DEC.5/10/98 de la CEDEAO; les résultats des travaux de la réunion des ministres, première réunion extraordinaire $d u$ Conseil d'Orientation du Programme
Régional Parc W/ECOPAS, tenue à Cotonou (République du Bénin) le 26 février 2004 et l'acte $n^{\circ}$ 20/87-UDEAC-475 portant adoption de l'accord de création de la Communauté Economique du Bétail, de la Viande et des Ressources Halieutiques en UDEAC dont le siège est fixé à N'DJAMENA.

Les différents pays de l'Afrique de l'Ouest ont procédé progressivement à une codification de la législation pastorale. A travers cet exercice, il s'agit d'ordonner les règles relatives à l'activité pastorale avec une volonté de rationalisation (Ly, 2004). Les différents Codes pastoraux adoptés ont organisé d'abord la gestion du pâturage en insistant sur sa définition d'une part et d'autre part aux conditions d'accès. Mais, on constate que la notion de pâturage est définie par chaque pays selon ses propres critères. Cette méthode ne facilite pas la cohésion d'ensemble des législations pastorales. Après cette définition, les législations déterminent les conditions d'accès à cet espace, la difficulté étant surtout liée au fait que le pastoralisme se caractérise par la mobilité des animaux et des éleveurs. La question de l'hydraulique pastorale est également intégrée dans ces textes. Dans ces différents codes relatifs à l'eau, les cas de concurrence entre différents types d'utilisations d'eau ont été prévus. Malgré ce nombre élevé de textes législatifs et réglementaires, il n'en demeure pas moins que les lois pastorales doivent être mieux harmonisées avec les codes de l'eau sur de nombreux points, particulièrement sur la question de l'accès aux ressources.

\section{SYSTÈME \\ TRANSHUMANT \\ Pôle humain}

L'élevage transhumant au Bénin est effectué essentiellement par les peuls nationaux et internationaux dont l'activité principale originelle est l'élevage. L'âge moyen de ces derniers est de 32 ans. Mais, les enfants sont utilisés pour conduire les troupeaux sur de courte voire moyenne 
distance. Ce sont les enfants qui, dès leur adolescence, prennent la relève du père pour perpétuer ce modes d'élevage. Ce qui fait que pour la totalité des cas, ce sont des illettrés (Lesse, 2009).

\section{Pôle animal}

Deux groupes de bovins sont exploités en République du Bénin ; les taurins et les zébus. Les races locales sont la race lagunaire, la race Somba, la race Borgou et la race Pabli. Les races importées (Les Zébus ou Bos indicus) sont la race Bororo ou Mbororo, la race Djelli., la race Bounadji ou White fulani et la race Goudali de Sokoto. La taille moyenne d'un troupeau varie en fonction des groupes socioprofessionnels qui définissent le mode d'élevage (Lesse, 2009 ; Houinato et al., 2000). L'effectif moyen par troupeau, varie de 55 à 107 selon ces auteurs. Ces valeurs peuvent s'expliquer par le fait que cette zone soudano-guinéenne est propice à cette activité. On observe aussi des ovins et les caprins au sein des troupeaux transhumants (Lesse, 2009). Dans les troupeaux Peulhs, les vaches représentent généralement plus de $65 \%$ du cheptel, grâce a la production de lait (Lesse, 2009). Le reste est constitué de veaux (19\%), de taureaux $(6 \%)$ puis de taurillons et de génisses $(10 \%)$.

\section{Gestion de l'espace et infrastructures d'élevage}

Le terroir étant soumis à une dynamique de changements, il évolue progressivement en défaveur de l'existence d'espaces pâturables. Les couloirs de passage sont réduits, les aires de pâturage sont désormais occupées par les champs et l'érosion du sol se fait de plus en plus sentir (Sounkere, 2003, Lesse, 2009). La transformation qui s'observe aujourd'hui sur l'espace est la résultante des techniques utilisées par les populations dans l'exploitation du milieu. De ce fait, on assiste à la dégradation de l'espace. Cette dégradation selon de Haan (1992) se subdivise en quatre groupes principaux : l'épuisement des sols en matière organique et nutriments, la dégradation physique des sols superficiels, l'érosion des sols et la dégradation du couvert végétal. Les ligneux occupent une place importante dans l'alimentation des bovins surtout en saison sèche où ils sont émondés. Les ligneux comme Afzelia africana, Khaya senegalensis et Pterocarpus erinaceus ont été cités comme étant des arbres fourragers de la saison sèche (Houinato, 2001 ; Brisso et al., 2007 ; Ahoudji, 2009). De la même façon, Wala et al (2005) ont cité Parkia biglobosa et Elaeis guineensis comme espèces ligneuses exploitées par les populations pendant la saison sèche. Mais, d'autres ligneux sont aussi émondés au Bénin. Il s'agit du Bombax costatum et Daniellia oliveri. D'après les éleveurs, Afzelia africana, Khaya senegalensis et Pterocarpus erinaceus se font de plus en plus rare (Ahoudji, 2009). Les mêmes espèces sont exploitées chaque année, ce qui empêche leur développement (Brisso et al., 2007). Houinato (2001) a signalé que les espèces telles que Khaya Senegalensis, Pterocarpus erinaceus, Afzelia africana et Daniellia oliveri qui subissent ces coupes régulières de leur frondaison, finissent à ne plus fructifier, ce qui compromet toute possibilité de leur régénération naturelle.

Axes de transhumance

A l'issue des débats et des échanges fructueux liés à la réunion des ministres, première réunion extraordinaire du Conseil d'Orientation du Programme Régional Parc $\mathrm{W}$, tenue à Cotonou (République du Bénin) le 26 février 2004, cinq (05) axes sont retenus et confirmés dans le statut officiel de la transhumance dans l'espace CEDEAO (Djohy, 2010). Ces axes de transhumance CEDEAO sont :

Axe 1 : Fada N'Gourma - Pama Porga - vers Togo ou Tanguiéta - Natitingou - Djougou -Bassila;

Axe 2 : (a) Sebba - Kantchari Diapaga - Namounou - Pagou - Porga - vers Togo ou Tanguiéta - Natitingou - Djougou Bassila; 
(b) Torodi - Makalondi - Kantchari Diapaga - Namounou - Pagou - Porga - vers Togo ou Tanguiéta - Natitingou - Djougou Bassila.

Axe 3 : Say - Tamou - Botou TapoaDjerma - Kotchari - Kondio - Kérémou - Toura - Goumori - Kerou - Vers zone d'accueil Alibori supérieur.

Axe 4 : Say - Kirtachi - Boumba Monsey - Karimama - Karigui -vers zone d'accueil Goungoun.

Axe 5 : Birni N'Gaouré - Dosso - Gaya - Malanville - Guéné - vers Goungoun.

L'entrée au Bénin se fait pour les éleveurs (avec leurs troupeaux) en provenance de pays limitrophes à partir des portes indiquées selon FAO (2012) comme suit :

(Niger): Malanville (officiellement, conformément à la loi $\mathrm{N}^{\circ}$ 87-013 du 21 septembre 1987), mais existence d'une multitude de portes d'entrée non autorisées ;

Burkina Faso : Porga ;

Togo : Atomey et Lanta ;

Nigeria: Waria, Kaboua, Toui, Ilikimou et Gbanago.

Une fois en territoire béninois, les zones de concentration des nationaux transhumants correspondent aux points terminaux des itinéraires et se répartissent comme suit (FAO, 2012) :

Borgou (Arrondissement d'Angaradebou, triangle Waria-Boukovo-Malété)

Atacora (Bassila, triangle Tanguiéta

Natitingou-Djougou)

Zou (Triangle Toui-Kilibo-Djègbé)

Mono (Le long des feuves Mono et Kouffo

sur $5 \mathrm{Km}$ de large et du côté Ouest

Ouémé (Dogo)

Atlantique (autour de Hinvi, Agon, Assagota, Koundokpoé)

Beaucoup de travaux ont été effectués sur les axes de transhumance au Bénin. Dans les terroirs riverains du parc W. Au total, neufs (09) couloirs de transhumance sont empruntés respectivement par les éleveurs nationaux et étrangers dans les terroirs riverains du parc $\mathrm{W}$ (Figure 1). Les aires d'accueil de ces troupeaux transhumants locaux sont entre autres : l'Alibori; la forêt classée de l'Alibori Supérieur, Kalalé, le Parc W, les forêts de la Sota, de Warimaro et le Parc Penjari. Les transhumants définissent leurs itinéraires en fonction des campagnes de transhumance (CSAO/OCDE, 2007). Ils sont ainsi variables en fonction des saisons et des situations socio-économiques locale. Ce qui fait que ces axes ne sont pas toujours effectivement utilisés.

\section{CONTRAINTES LIEES A L'ALIMENTATION}

Pour jouer efficacement ce rôle important (économique, social et culturel), la productivité des animaux doit augmenter. L'importance de cette activité est liée à son apport au PIB, sa contribution à la sécurité alimentaire et nutritionnelle des populations rurales. Or l'une des contraintes majeures qui freinent le développement des systèmes actuels de production animale en Afrique reste et demeure l'alimentation, notamment pendant les périodes de saison sèche (Xandé, 1989; Adandédjan, 1999). En Afrique, l'élevage des ruminants et en particulier celui des bovins se base sur l'utilisation extensive des pâturages naturels (Akpo et al., 1999). L'augmentation de la population, le renchérissement des prix de certains produits agricoles comme le coton, l'amélioration et l'organisation de la filière coton ont provoqué une ruée vers les terres fertiles jusque-là épargner des pratiques agricoles (Sinsin, 1993). Aussi, l'utilisation de moyens de production améliorés (traction animale) ou modernes a favorisé la conquête d'immenses espaces naturels à des fins agricoles, parfois au détriment des aires protégées et des aires de pâturage. Ce qui fait que les écosystèmes naturels subissent de fortes dégradations. 


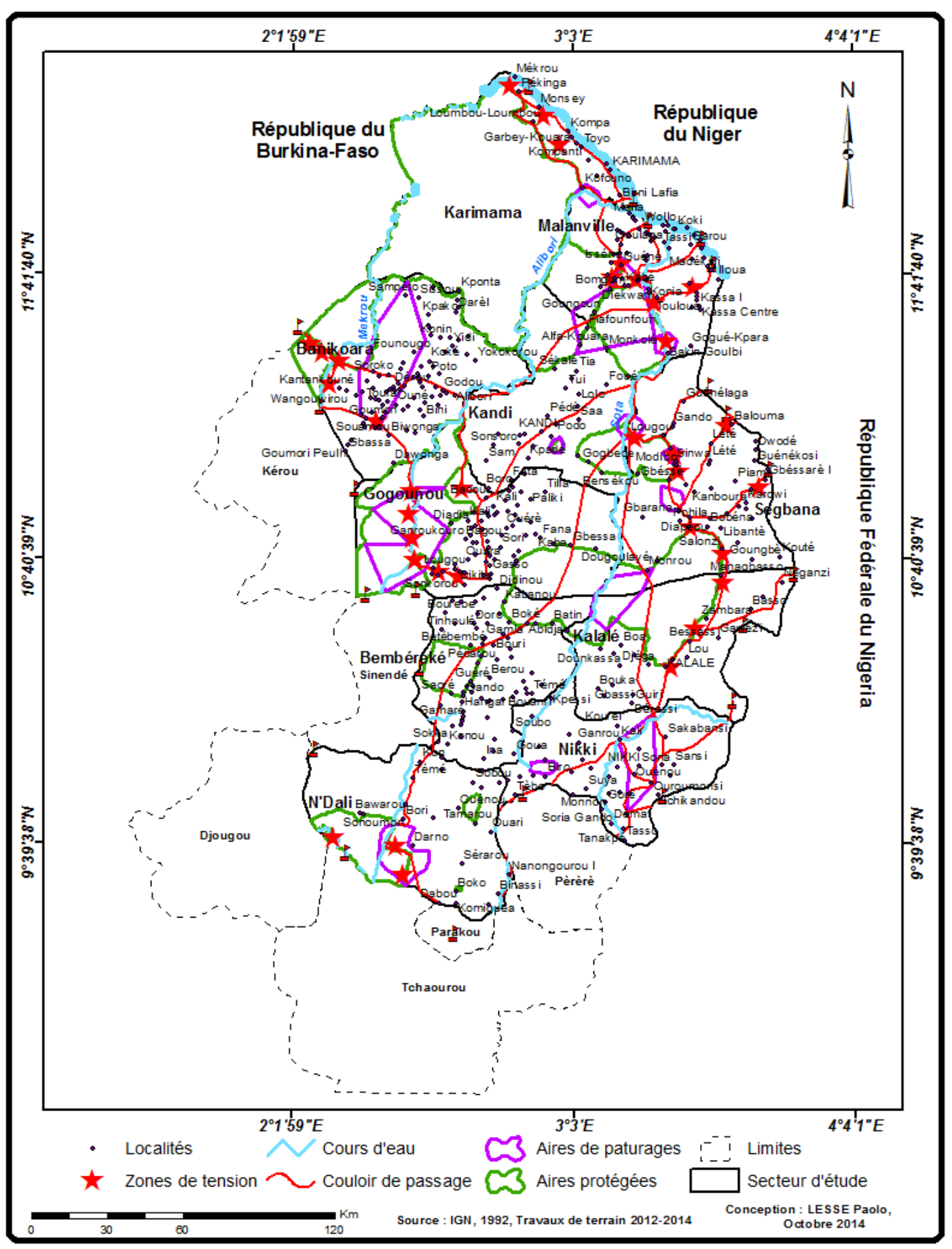

Figure 1 : Carte de transhumance dans les départements du Borgou et de l'Alibori. 
Kagone et al. (2006) et Convers (2002) ont signalé alors que les aires protégées subissent des incursions des transhumants. Donc pour une gestion efficiente des parcours naturels, Djenontin (2010) propose l'intégration des éleveurs de ruminants dans les programmes d'aménagement forestier et dans la gestion des terroirs. Pour cet auteur, les stratégies pour l'utilisation des parcours naturels pour l'alimentation des troupeaux bovins doivent tenir compte des facteurs climatiques et anthropiques.

La variabilité spatio-temporelle de la pluviosité dans ces milieux rend aléatoire la disponibilité des ressources alimentaires pour les animaux notamment en saison sèche. Dans ce type d'élevage où la végétation naturelle est de loin la ressource fourragère la plus importante, chaque animal a besoin de plusieurs hectares d'espace, ce qui contraste avec les besoins de la production agricole. La conséquence directe est le surpâturage qui induit la chute de la productivité des parcours. Ainsi, la biomasse herbacée varie dans le temps suivant les conditions pluviométriques (Sawadogo et al., 2005) et dans l'espace suivant la nature du substrat édaphique liée à la situation topographique (Toko et Sinsin, 2011a ; Sinsin, 1993). Si les pâtures ne s'effectuent pas dans des zones classées et que les couloirs de passage ne sont pas respectés de même que les périodes de transhumance, on assiste très souvent à des dégâts sur les cultures, ce qui débouche sur des conflits parfois violents entre agriculteurs et éleveurs.

\section{CONTRAINTES LIEES AUX VARIABILITES CLIMATIQUES}

Plusieurs études ont montré que chaque année, la quantité de pluies influence celle de la biomasse herbacée tandis que la pâture modifie la flore (Achard et Inalher, 1998 ; Breman et al., 1997). Au Bénin, notamment dans la partie septentrionale qui abrite la plus grande part du cheptel du pays, les travaux de Donou et al., (2008) ont révélé que les contraintes climatiques ont entraîné une diminution de matières sèches au niveau des pâturages. En conséquence, les éleveurs migrent avec leurs troupeaux vers la partie méridionale où ils accroissent les pressions sur les ressources naturelles. On a noté une intensification des sécheresses qui se sont produites pendant la même période, notamment dans les années 1970 et 1980 (MEHU, 2001). Ogouwalé (2006) prévoit à l'horizon 2050 une hausse des températures de $+1{ }^{\circ} \mathrm{C}$ à $+3{ }^{\circ} \mathrm{C}$ et une perturbation du régime pluviométrique. $\mathrm{Si}$ ce scénario se confirme, il induira des stress supplémentaires pour les espèces fourragères pouvant entraîner des modifications sur les écosystèmes, la raréfaction voire la disparition de cours et plans d'eau et des pâturages. Certains auteurs ont montré, qu'en plus des modifications dans les précipitations moyennes globales, il pourrait avoir des changements plus prononcés dans les caractéristiques des précipitations locales et régionales dues au réchauffement climatique. Par exemple, Trenberth et al. (2003) ont émis l'hypothèse qu'en moyenne, les précipitations seront moins fréquentes mais plus intenses quand elles se produiront, impliquant de ce fait une grande fréquence d'extrêmes inondations et sécheresses.

\section{CONTRAINTES LIEES AUX CONFLITS}

Dans ce nouveau contexte où les variabilités climatiques se sont ajoutées, la transhumance peut être considérée comme une forme d'adaptation à ces environnements et de valorisation des complémentarités écologiques entre zones sahéliennes et régions soudaniennes. En définitive, il s'agit d'un système d'élevage fondé sur une stratégie de gestion opportuniste des ressources pastorales mais écologiquement viable, qui a permis à des communautés de pasteurs, les Peuls, les Touaregs et les Maures notamment, de survivre aux grandes crises éco-climatiques qui secouent périodiquement les pays 
sahéliens. Chaque année, les mouvements de transhumance sont marqués par des tracasseries administratives et de graves incidents dans les zones d'accueil, liés au nonrespect des réglementations nationales et régionales, aux dégâts occasionnés aux champs et/ou aux récoltes, à l'exploitation pastorale des aires protégées, aux pertes d'animaux, etc. Ce climat de tension permanente entre les éleveurs transhumants et les populations des zones d'accueil entraîne des conflits, parfois meurtriers (Akpaki, 2002). Au cours de leur transit, les transhumants transfrontaliers entretiennent tant des rapports conflictuels que de bon voisinage avec les élus locaux et les leaders d'éleveurs. Au nombre des causes majeures de conflits, l'occupation des couloirs par les champs et la pâture des animaux dans ces derniers se retrouvent en bonne place. En effet, chacun des acteurs selon sa position revendique des droits de propriété relatifs aux pâturages, aux espaces cultivés, à certains couverts ligneux ou aux plans d'eau (Camara, 2002). Les conflits entre agriculteurs et éleveurs sont omniprésents dans toutes les discussions de terrain et les mécanismes de gestion étatique (comité de transhumance) sont peu équitables et inefficace. Cette forte pression sur les ressources va considérablement modifier les rapports qu'entretiennent les acteurs du monde rural et particulièrement les rapports entre les éleveurs et les agriculteurs. Ces rapports sont fortement imprégnés de conflits violents parfois collectifs. Ces relations conflictuelles entre ces deux communautés résultent de la concurrence de plus en plus vive entre le pastoralisme et l'agriculture, car ces deux activités reposent sur l'utilisation extensive de l'espace rural.

\section{CONTRAINTES LIEES AUX TEXTES REGLEMENTAIRES}

La détermination des pays de la sousrégion à améliorer les conditions de la transhumance transfrontalière est notée à travers ces textes. A cet effet, ils se sont dotés, chacun, d'un dispositif législatif et règlementaire approprié. Toutefois, certaines pratiques de contrôle et/ou de gestion de la transhumance transfrontalière ne sont pas toujours conformes aux règlements en vigueur dans les pays et au niveau de la CEDEAO. En effet, la Décision A/DEC.5/10/98 de la CEDEAO qui définit la transhumance et comporte d'importantes dispositions qui visent à atténuer les tensions sociales et prévenir les conflits liés à la transhumance transfrontalière (fixation piste de transhumance, période d'entrée et de sortie ; les zones d'accueil dans chaque pays par chaque membre de la CEDAO et en ce qui concerne les éleveurs, la détention du certificat international de transhumance, la garde des animaux transhumants, la conduite des animaux uniquement sur les pistes de transhumance) n'est pas appliquée de façon systématique dans les pays membres de cette institution. Ainsi, la libre circulation des personnes et des biens, ainsi que l'accès aux marchés porteurs dans les différents pays resteront entravés, si une volonté politique de concertation et de mise en œuvre ne se dégage pas. Egalement, une vulgarisation de ces textes n'est pas faite et ne tient pas compte des réels problèmes des éleveurs. Dans ce contexte, ces textes doivent être revus car ils sont désuets, équivoques et non connus des principaux acteurs (Djohy, 2010). Les structures d'intervention fonctionnent sur du top-down, proposant des solutions, peu adaptées aux réalités locales.

Les collectivités locales, avec leur modicité financière, contournent la réglementation et se donnent des marges leur permettant d'accepter clandestinement les transhumants étrangers. Tous ces facteurs rendent le climat social plus conflictuel entre acteurs du monde agricole et pastoral. 


\section{CONTRAINTES LIEES AU SYSTEME D'ELEVAGE}

La transhumance qui était un fait socioculturel dans la région du Parc du W revêt maintenant une nouvelle forme et possède de nouveaux objectifs (fuir les conflits avec les paysans agriculteurs, faire pâturer les animaux délibérément et de façon illégale et éviter à tout prix le forestier). L'élevage transhumant s'est alors adapté et n'obéit plus au mouvement pendulaire avec pour départ la fin de la saison pluvieuse et le retour au début de la saison pluvieuse comme décrit par Sinsin (1985). Les éleveurs, par adaptation à l'environnement, continuent par pratiquer un système présenté par de Haan (1992) comme condamné car la gestion du bétail est dominée par une exploitation extensive de l'environnement à la recherche d'eau et de pâturages et le désir d'avoir un troupeau assez vaste pour pouvoir transmettre une partie à la descendance. Cette nouvelle forme de transhumance, à l'analyse, compliquera les relations entre l'administration forestière et les éleveurs, la pratique de l'élevage et la sauvegarde des aires protégées deviennent complexe. Selon Sounkere (2003), Les forestiers accepteront par exemple le principe des coupes contrôlées de paille et de fourrage dans certains secteurs des aires protégées. La zone tampon du Parc W pourrait être également utilisée à cet effet.

\section{CONTRAINTES LIEES AUX INFRASTRUCTURES PASTORALES}

Les infrastructures pastorales sont dégradées et insuffisantes (Djohy ,2010). Les infrastructures hydrauliques auxquelles s'associent les aires de pâturage ont permis d'avoir des communes et des arrondissements de concentration du bétail. Ces infrastructures sont quasiment inexistantes au sud du Bénin. Mais les aires de pâturage sont dans un état de dégradation avancée et ceci explique la tendance des éleveurs à exploiter les prairies et les savanes herbeuses dans les forêts classées et le Parc W. Les aires de pâturage apparaissent alors insuffisantes et justifient les mouvements saisonniers des troupeaux et des éleveurs et parfois leur émigration de leur commune d'origine. Dans le département de l'Alibori, les infrastructures marchandes et sanitaires ont permis un accroissement du cheptel et la mise en place d'une filière de produits d'élevage. Un renforcement de ces infrastructures sanitaires et marchandes participerait de la promotion de l'élevage dans les communes de ce département.

\section{CONTRAINTES LIEES A LA DYNAMIQUE DES AXES DE TRANSHUMANCE}

Les éleveurs rencontrent actuellement beaucoup de difficulté pour nourrir leurs animaux. Ces difficultés sont dues aux différents changements qu'ils observent dans l'environnement aux cours de ces dernières années. Ces changements pourraient avoir plusieurs causes dont la disponibilité de l'espace pâturable en saison pluvieuse qui est de plus en plus réduite (Sounkere, 2003). A cela s'ajoute l'obstruction des couloirs par les champs, le tarissement un peu plus rapide des mares et des cours d'eau (Lesse, 2011) et enfin d'après Paris (2002), l'émergence de l'élevage agropastoral des agriculteurs qui entre en concurrence avec l'élevage transhumant. Ce dernier affecte les relations agriculteurs - éleveurs, car minimisant les complémentarités et exacerbant la concurrence dans l'utilisation de l'espace et des ressources. Ce qui fait que la plupart des transhumants ont changé leurs itinéraires et d'autres prennent la direction des aires protégées pour garantir la sécurité alimentaire aux animaux sachant qu'ils courent des risques. Cette pratique a des inconvénients tant sur l'aire protégée que sur les transhumants et leurs troupeaux. A ce propos, Sounkere (2003) donnait quelques risques en ce qui concerne le parc $\mathrm{W}$ à savoir : L'empoisonnement des grands fauves par les 
éleveurs (cas du lion Panthera leo) dans le Parc du W; les risques de transmission de maladies (contact des bovidés domestiques avec ceux sauvages (buffles)) ; le braconnage effectué par les éleveurs autant pour la vente que pour la consommation sur place (Faaki, 1996), et la concurrence au niveau des pâturages entre les ruminants sauvages et domestiques.

\section{CONCLUSION}

La transhumance est un système d'élevage qui occupe une place importante dans les systèmes d'élevage de la sous-région et du Bénin en particulier. La présente étude a permis de faire un bilan des études réalisées sur ce système d'élevage et d'en ressortir ses différentes contraintes. Il en ressort que la transhumance est confrontée à des problèmes d'alimentation, de variabilités climatiques, des textes règlementaires, de conflits et de disponibilités des infrastructures pastorales. Il ressort également que la transhumance demeure sous la forte influence des systèmes de culture surtout pendant la sécheresse. Ce qui fait que la conduite de ce système a changé et tourné vers l'exploitation des aires protégées. En outre, les éleveurs ont adopté ce mode de transhumance actuelle pour exploiter le croît annuel des animaux ayant lieu en période de pluie. Ce croît ayant baissé ces dernières années, alors les éleveurs trouvent dans la visite des aires protégées en cette période, la recrudescence de l'effectif du cheptel.

\section{CONFLIT D'INTERETS}

Les auteurs déclarent qu'ils n'ont aucun conflit d'intérêts dans le cadre du présent manuscrit.

\section{CONTRIBUTIONS DES AUTEURS}

PL est intervenu dans toutes les phases de l'étude. Il s'agit de la conception de l'étude, la collecte des données, le dépouillement, le traitement et l'analyse des données et la rédaction du présent manuscrit ;
MRBH est intervenu dans la conception de l'étude, le dépouillement, le traitement et l'analyse des données et la rédaction du présent manuscrit; JD est intervenu dans la conception de l'étude, le dépouillement, le traitement et l'analyse des données et la rédaction $\mathrm{du}$ présent manuscrit;HD est intervenu dans le traitement des données et dans la correction du manuscrit; BY est intervenu dans le traitement des données, dans la correction du manuscrit; IT est intervenu dans le traitement des données, dans la correction du manuscrit; BT est intervenu dans le traitement des données, dans la correction du manuscrit; BS est intervenu dans la correction du manuscrit.

\section{REMERCIEMENTS}

A la fin de ce travail, les auteurs tiennent à remercier TOKO Nourou et AKADIRI Farrid pour leur soutien dans la collecte des données.

\section{REFERENCES}

Achard F, Inalher I. 1998. Evaluation de l'effet des différentes techniques de réhabilitation des sols encroûtés sur la végétation des jachères de Banizoumbou. p.47.

Adandedjan CC. 1999. L'Agroforesterie pour une Utilisation et une Gestion Durable des Terres et des Ressources Naturelles en Afrique du Sub Saharienne Humide. ICRAF.DSO : Kumassi, Ghana ; 8.

Ahoudji M. 2009. Transhumance et changement climatique: Caractérisation des populations des principaux LF dans les terroirs riverains du parc $\mathrm{W}$. Thèse d'Ingénieur agronome, FSA/UAC, Université d'Abomey-Calavi, Benin, p. 90 .

Akpaki J. 2002. «Ackerbauern und mobile Tierhalter in zentral- und Nord-Benin, Landnutzungskonflikte und Landesentwicklung» Berlin; Freie Universität Berlin. p.152

Akpo E, Grousis M, Bada F, Pontanier R, Floret C. 1999. Effet du couvert ligneux 
sur la structure de la végétation herbacée de jachères soudaniennes. Sécheresse, 0 (4): 253-61.

Atchy AA. 1976. Contribution à l'étude de la transhumance en république populaire de la transhumance en république populaire du Bénin. Thèse de Doctorat, P. 109.

Bernus E. 1995. Pasteurs face à la sécheresse: rebondir ou disparaître? ; IRD; p. 5.

Breman H, Cissé AM. 1997. Dynamics of saheliean pastures in relation to drought and grazing. Oecologigia (Berl.), 28 : 301-315.

Brisso N, Houinato M, Adandédjan C, Sinsin B. 2007. Dry season woody fooder productivity in savannas. Ghanaian Journal of Animal Science, 2,3(1): 181185.

Camara L. 2002. Savoirs, besoins et institutions territoriales dans les périphéries du parc w: typologies d'acteurs et configurations conflictuelles a partir de six villages-cibles. Recherche en Géographie Humaine- Volet « Dynamiques d'acteurs, conflictualité, concertation ». Université de L'AquilaItalie, P. 161.

CEDEAO. 2009. Note adoptée d'orientation pour le développement de l'élevage dans l'espace CEDEAO, p.6.

Convers A. 2002. Etat des lieux spatialisé et quantitatif de la transhumance dans la zone périphérique d'influence du parc national du W (Niger). Rapport de stage. DESS. Université Montpellier II, Cirademvt, p. 99.

De Haan L. 1992. Rapports entre agriculteurs et éleveurs au Nord-Bénin: écologie et interdépendance transformée. Rapport final. Tome 1 Rapport principal. FSA/UAC, p. 209.

Djenontin JA. 2010. Dynamique des stratégies et pratiques d'utilisation des parcours naturels pour l'alimentation des troupeaux bovins au Nord- Est du Bénin. Thèse de doctorat, Université d'AbomeyCalavi, Bénin. p.203.
Djohy G. 2010. Une analyse des dynamiques sociopolitiques et organisationnelles d'adaptation des éleveurs transhumants dans l'Alibori (Nord - Bénin). Thèse d'Ingénieur agronome, FA/UP, Université de Parakou, Benin p. 122.

Donou B, Ogouwalé E, Yabi I, Boko M. 2008. Contraintes climatiques et pression sur les pâturages dans le Département des collines (République du Bénin). Revue de Géographie du Bénin Université d'Abomey-Calavi (Bénin), 3: 61-75.

Dugué P, Jouve Ph. 2003. Organisation spatial et gestion des resources et des territoirres ruraux. Actes du colloque international, 25-27 février 2003, Monpellier, France.

Eboh EC, Oji KO, Oji OG, Amakom U, Ujah OC. 2008. Towards the ECOWAS Common Agricultural Policy Framework: Nigeria Case Study and Regional Analysis. African Institute for Applied Economics: Enugu, Nigeria. P. 197.

Faaki AV. 1996. Analyse des formes d'occupation concurrentes des terres dans la zone tampon aux aires protégées: cas du périmètre de Sampéto dans la souspréfecture de Banikoara au Nord-Bénin. Thèse d'Ingénieur agronome, FSA/UAC, Université d'Abomey-Calavi, Benin, p. 113.

FAO. 2010. Guide d'application au niveau intermédiaire. Programme d'analyse socio-économique selon le genre, p.120.

FAOSTAT. 2014. Countrystat Bénin, www.countrystatbenin.org consulté le 2 Août 2014

FAO. 2012. La transhumance transfrontalière en Afrique de l'Ouest: proposition de plan d'action. Rapport d'étude, p.146.

Houinato M. 2001. Phytosociologie, écologie, productivité et capacité de charge des formations végétales pâturées dans la région des Monts-kouffé (Bénin). Thèse de doctorat en sciences agronomiques. Université de bruxelles, Belgiques, p. 219. 
Houinato M, Sinsin B. 2000. La pression agropastorale sur la zone riveraine de la réserve de biosphère de la Pendjari. Tropicultura, 18(3): 112-117.

Kagoné H, Toutain B, Dulieu D, Houinato M, Boureima A, Nocker U. 2006. Pastoralisme et Aires protégées en Afrique de l'Ouest: Du conflit à la gestion concertée de la transhumance transfrontalière dans la région du parc $\mathrm{W}$ (Bénin, Burkina-Faso, Niger). Bull Animal. Hlth. Production. Afr, 54: 43-52.

Lesse P. 2009. Transhumance et changement climatique: Productivité et capacité de charge des pâturages naturels des terroirs riverains de la réserve de biosphère transfrontalier du W (Bénin), Thèse d'Ingénieur agronome, FSA/UAC, Université d'Abomey-Calavi, Benin, p. 107.

Lesse P. 2011. Analyse de la gestion pastorale et de l'adaptation des éleveurs transhumants face aux variabilités climatiques dans les communes riveraines de la Réserve de Biosphère Transfrontalière du W (Bénin), thèse DEA Université d'Abomey-Calavi, Bénin, p. 102.

Lhoste Ph, Dollé V, Rousseau J, Soltner D. 1999. Manuel de Zootechnie des Régions chaudes. Les Systèmes d"Élevage. (Collection et précis deélevage). Ministère de la Coopération: Paris p. 288.

Lombard J. 1957. Quelques notes sur les Peuls au Dahomey, Bull. de l'information et Correspond. de l'IFAN, 73: 5-7.

LYI. 2004. Essai de présentation des tendances d'évolution du droit pastoral en Afrique de l'ouest: Burkina-Faso, Guinée, Mali, Mauritanie, Niger, Sénéga,1 p. 36.

Ministère de l'Environement, de l'Habitat et de l'Urbanisme. 2001. Communication Nationale Initiale du Bénin sur les Changements Climatiques. MEHU: Cotonou. p. 76.
Ogouwalé E. 2006. Changements climatiques dans le Bénin méridional et central: indicateurs, scénarios et prospective de la sécurité alimentaire. Thèse de Doctorat Unique, Université d'Abomey-Calavi, Bénin, p. 302.

Paris A. 2002. Etat des lieux quantitatif et spatialisé de la transhumance en périphérie du Parc du W (Burkina-Faso). Rapport de stage. DESS. Université Montpellier II, CIRAD-EMVT, p. 71.

Pecaud M.G. 1912. L'élevage des Animaux Domestiques au Dahomey Gorée, Imprimé du Gouvernement Général de l'A.OF, p.26.

Renard M. 2010. Vulnérabilités des populations pratiquant l'élevage et gestion des ressources naturelles pastorales de la commune de Djougou au Bénin. Mémoire de Master 1 de Géographie. Université Toulouse II Le Mirail, p. 68.

Sawadogo L, Tiveau D, Nygard R. 2005. Influence of selective tree cutting, livestock and prescribed fire on herbaceous biomass in the savannah woodlands of Burkina Faso, West Africa. Agriculture, Ecosystems and Environment, 105: 335-345.

Sinsin B. 1985. Contribution à l'utilisation rationnelle des ressources naturelles : impact des activités anthropiques (braconnage et activités agropastorales). Thèse d'Ingénieur agronome, FSA/UAC, Université d'Abomey-Calavi, Benin, p. 172.

Sinsin B. 1993. Phytosociologie, écologie, valeur pastorale, production et capacité de charge des pâturages naturels du périmètre Nikki-Kalalé au Nord-Bénin. Thèse de doctorat en sciences agronomiques. Université de bruxelles, Belgiques. P. 390.

Sounkéré K. 2003. Analyse des formes d'utilisation de l'espace dans les terroirs agropastoraux de la périphérie du parc national du $\mathrm{W}$ : cas de la commune de Karimama. Thèse d'Ingénieur agronome 
FSA/UAC, Université d'Abomey-Calavi, Benin, p.82.

Toko I, Sinsin B. 2011a. Facteurs déterminant la variabilité spatiale de la biomasse herbacée dans la zone soudano-guinéenne du Bénin. Int. J. Biol. Chem. Sci, 5(3): 930-943. www.ajol.info/index.php/ijbcs/ article/viewFile/72180/61117

Trenberth KE, Dai A, Rasmussen RM, Parsons, DB. 2003. The changing character of precipitation. Bulletin of the American Meteorological Society, 84(9): 1205-1217.

Wala K, Sinsin B, Guelly KA, Kokou K, Akpagana K. 2005. Typologie et structure des parcs agro-forestiers dans la préfecture de Doufelgou (Togo). Sécheresse, 16(3) : 209-216.

Xandé A, Garcia Trujillon R, Caceres O. 1989. Feed of the humid tropics: West Indies. In Recommended Allowances and Feed Tables, Jarrige R (ed). Ruminant Nutrition : Londres; 347-363.

Zekpete E. 2009. Transhumance et changement climatique: utilisation des outils d'aide à la décision dans la gestion durable des ressources des écosystèmes agropastoraux soudano-sahéliens du Nord-Bénin. Thèse DESS, Université d'Abomey-Calavi, Bénin, p.112. 